\title{
Préface de la deuxième édition anglaise
}

Lors de la préparation de cette seconde édition, nous avons été très conscients de notre engagement initial à refléter les développements les plus récents de l'emploi des méthodes de spectroscopie et de diffraction X, pas seulement pour analyser les éléments et les groupes présents, mais aussi pour établir l'arrangement des atomes constituants. Ce but reste notre objectif majeur, en mettant l'accent sur la manière dont ces approches fournissent une meilleure compréhension de la structure moléculaire, et aussi, bien sûr, comment leur application aide les étudiants de première année à apprendre et à développer leur habileté en analyse structurelle. Comme précédemment, nous avons choisi d'inclure des chapitres sur la spectrométrie de masse et la diffraction ( $X$, neutrons et électrons), aussi bien que sur les méthodes spectroscopiques (IR, UV, RMN) pour illustrer les informations cruciales qu'elles apportent à l'analyse structurelle (par exemple sur les masses moléculaires, sur les formules empiriques et structurelles, en deux et en trois dimensions).

En révisant ce texte et son contenu, nous nous sommes efforcés de fournir des occasions d'approfondissement, à l'aide d'exercices résolus, de suggestions de travail personnel, et de questions à choix multiples en ligne. Notre but est d'encourager la pratique des manipulations de base, avant de progresser avec des exemples plus avancés.

Nous avons ajouté des nouvelles sections, comme les spectroscopies Raman, micro-onde et infrarouge, la diffraction des neutrons et celle des électrons, à côté de celle des $X$. Nous avons aussi amélioré notre traitement des méthodologies basées sur l'emploi des technologies informatiques. En particulier, en traitant la spectroscopie IR et Raman, nous avons mis plus de poids sur les populations des états énergétiques, sur les règles de sélection et sur les transitions, sur les paramètres géométriques comme les longueurs de liaison, et sur ceux utilisés dans la reconnaissance d'une structure. Nous avons aussi porté une attention particulière aux effets que la symétrie moléculaire, la taille et la phase jouent en déterminant les sortes d'informations disponibles, et comment employer une méthode spécifique de manière optimale.

Le domaine d'application de ces techniques a été étendu, en particulier vers les systèmes biologiques et médicaux, et par exemple vers le développement de l'imagerie médicale par résonance magnétique. Nous avons aussi tenu compte de la demande de modifier l'instrumentation médicale, jugée trop coûteuse et impossible à opérer dans un cabinet médical ordinaire. II en est de même des spectromètres haut de gamme ou même de ceux appelés à fonctionner dans un environnement hostile (par exemple sur d'autres planètes). Mais notre principal 
souci reste d'aider les étudiants dans leur capacité de comprendre et d'interpréter les signaux que leur apportent les instruments. Nous sommes persuadés que la maîtrise de ces problèmes conduit le lecteur au cœur des méthodes employées, et le stimulera à entreprendre des études et des applications plus avancées.

Nous remercions spécialement ceux qui nous ont aidés en nous fournissant des commentaires utiles, ou des spectres, ou les deux, grâce à l'accès à leurs publications dans la littérature originale. Notre gratitude va également à nos collègues Ian Fairlamb, Brendan Keeley, Jason Lynam et Peter O'Brien, ainsi que John Moore et Derek Wann pour des discussions utiles. Nous sommes aussi reconnaissants envers Adrian Whitwood pour ses commentaires utiles, pour l'accès à ses publications et ses dossiers sur ordinateur, et pour son assistance on line. Nous aimerions encore remercier Lyndsay Muschamp et de Katie Scott, pour le soin et l'expertise qu'elles ont manifestés lors de la production des manuscrits, et à toute l'équipe du OUP, en particulier Alice Roberts, pour son expertise, sa patience et son appui.

S.B.D., B.C.G. et M.C.R.C.

York 2015 